\title{
Feasibility study of a single-use balloon-assisted robotic colonoscope in healthy volunteers
}

\section{다 (용}

\author{
Authors \\ Chi-Chung Foo', Wai-Keung Leung ${ }^{2}$, Thomas Ka-Luen Lui ${ }^{3}$, Jo Lai-Ken Cheung ${ }^{4}$, Kwok-Wai Lam ${ }^{4,5}$, Biji Sreedhar $^{4}$, \\ Chung-Kwong Yeung ${ }^{1,4}$
}

\section{Institutions}

1 Department of Surgery, Li Ka Shing Faculty of Medicine, The University of Hong Kong, Hong Kong SAR

2 Department of Medicine, Li Ka Shing Faculty of Medicine, The University of Hong Kong, Hong Kong SAR

3 Department of Medicine, Queen Mary Hospital, The University of Hong Kong, Hong Kong SAR

4 Bio-Medical Engineering (HK) Limited, Hong Kong SAR

5 Department of Orthopaedics and Traumatology, Li Ka Shing Faculty of Medicine, The University of Hong Kong, Hong Kong SAR

submitted 28.8.2020

accepted after revision 30.11 .2020

Bibliography

Endosc Int Open 2021; 09: E537-E542

DOI 10.1055/a-1352-3688

ISSN 2364-3722

(C) 2021. The Author(s).

This is an open access article published by Thieme under the terms of the Creative Commons Attribution-NonDerivative-NonCommercial License, permitting copying and reproduction so long as the original work is given appropriate credit. Contents may not be used for commercial purposes, or adapted, remixed, transformed or built upon. (https://creativecommons.org/licenses/by-nc-nd/4.0/)

Georg Thieme Verlag KG, Rüdigerstraße 14,

70469 Stuttgart, Germany

\section{Corresponding author}

Dr Chi-Chung Foo, MBBS, Department of Surgery, Li Ka Shing Faculty of Medicine, The University of Hong Kong, Pokfulam Road, Hong Kong SAR

ccfoo@hku.hk

\section{ABSTRACT}

Background and study aims Despite its widespread adoption, colonoscope still has its limitations. Advancement is often limited by the looping of colon. The isolation of SARS-CoV-2 in stool raises concern for the risk of disease transmission. A single-use robotic colonoscope, the NISInspire-C System, that features a balloon-suction anchorage mechanism was developed to address these.

Methods The NISInspire- $C$ balloons are designed to provide anchorage for straightening of the colon during advancement. Angulation at the bending section is tendonwire driven by servo mechanisms integrated into a robotic control console. This was a pilot, prospective trial to evaluate the safety and feasibility of this system. Healthy volunteers underwent examination with the NISInspire-C, followed by the conventional colonoscope. The procedure time, cecal intubation rates (CIR), complications, and level of pain were measured.

Results A total of 19 subjects underwent the examination. The cecal intubation rate was $89.5 \%(17 / 19)$ and the overall time-to-cecum was 26.3 minutes (SD: 17.9 mins). There were no procedure-related complications. Polyps were detected in seven of 19 (36.8\%) subjects during the NISInspire- $C$ procedure. Three more subjects were found to have adenomatous polyps with the conventional colonoscope. There was minimal variation in level of pain during the procedures with the two colonoscopes.

Conclusion The single-use robotic colonoscope NISInspire- $C$ is a safe and feasible alternative to the conventional colonoscope. Further technical refinement is needed to improve the CIR. This study was limited by its small sample size.

\section{Introduction}

Screening for colorectal cancer (CRC) remains the most important strategy for prevention of the disease. Conventional colonoscopy has been the method of choice and a gold standard for the last 40 years. The conventional colonoscopes require endoscopists to manually advance them to navigate inside the colon lumen. This is often accompanied with looping of the scope, which causes discomfort and pain and may hinder successful cecal intubation. Another increasing concern is the possibility of infectious disease transmission through reuse of colonoscopes along with the need for cleaning, disinfection, and maintenance costs to ensure optimal performance [1,2].

Various alternatives to conventional colonoscopes have been developed to address current deficiencies, including robotic-actuated colonoscopes. Here, we developed a single-use 
robotic colonoscope that features a balloon-suction anchorage mechanism. The balloons are designed to provide a firm but soft anchorage by physical means. That can help to undo loops and allow straightening of the colon for advancement of the colonoscope. The scope also is designed for single use, eliminating risks of cross-contamination between patients.

\section{Methods}

This was a pilot, prospective trial of a novel colonoscope, (Clinical Trial.gov Identifier: NCT03979690). The primary objective of this trial was to evaluate the safety and feasibility of the device. The study was approved by the Ethics Committee/Institution Review Board of the University of Hong Kong (IRB reference no. UW 18-560, UW 18-561). Two study sites, both located in a local hospital, and three investigators were involved in the study. All participants in the study were healthy volunteers who were willing to undergo a screening colonoscopy. The inclusion criteria were symptomatic adults indicated for elective colonoscopy or asymptomatic adults willing to undergo a colonoscopic screening for polyps or colorectal cancer, aged 40 to 77 years, who had not had a previous screening colonoscopy and were willing to provide signed informed consent. Each subject was on a low-residue diet 2 days before and a fluid diet 1 day before the colonoscopy procedure and received a conventional bowel lavage procedure using polyethylene glycol (PEG) solution (Klean-Prep, Helsinn Birex Pharmaceuticals Ltd, Dublin, Ireland) in split dosage. The cleanliness of the bowel preparation was classified according to the Boston Bowel Preparation Scale (BBPS) [3]. Each subject underwent colonoscopy using the NISInspire-C System, followed immediately by conventional colonoscopy. A complete examination was defined by cecal intubation. Terminal ileal intubation was optional. All endoscopy examinations using the NISInspire-C System were performed by designated experienced endoscopists with cecal intubation rates (CIRs) of over $95 \%$ [4]. Each designated endoscopist was previously trained on using the NISInspire-C System.

The NISInspire-C is a disposable balloon-assisted colonoscope, designed with a robotic bending mechanism that allows precise omni-directional bending of the colonoscope towards areas of interest for performing diagnostic or therapeutic procedures ( $\mathbf{F i g}$. 1a) [5]. The articulated bending section is 90 $\mathrm{mm}$ long near the distal portion of the colonoscope with a maximum mechanical angulation of 160 degrees in all directions. Angulation at this bending section is tendon wire-driven by servo mechanisms integrated into the robotic control console. The bending section is covered by a thin outer sleeve with a very low coefficient of friction for increasing the efficiency of advancement. A twin balloon-assisted anchoring mechanism is situated at the distal end when deployed can facilitate the straightening of the colon and undo loop formations. The twin-balloon anchorage mechanism is based on two elements: 1) two inflatable balloons that, once activated, create a partition in the colon lumen; and 2) a suction port that creates negative pressure between the partition to collapse the colon wall to the colonoscope, creating anchorage motion. In addition to the anchorage properties of the balloons for promoting successful cecal intubation, the balloons can also act as flanges to depress mucosal folds inside the colon thereby enhancing luminal examination during colonoscope withdrawal to improve lesion detection hidden from behind the mucosal folds (> Fig.1b). Advancement of the NISInspire-C inside the colon is illustrated in - Fig. 2. The colonoscope was equipped to perform the same basic functions as a conventional colonoscope, such as air $/ \mathrm{CO}_{2}$ insufflation and water irrigation/suction, and had a 2-mm working channel for biopsy or a surgical instrument, and a water jet for cleaning the camera lens. The NISInspire-C colonoscope has a full HD CMOS image sensor with a 2 mega-pixel resolution with a high dynamic range and a low signal-to-noise ratio output performance. The image sensor can capture highresolution video images at a frame rate of 30 frames per second. Based on this high-resolution imaging technique, realtime diagnostic observations as well as postoperative classification of the captured video frames or still images can be effectively performed. The design of the optical system employs a precision objective lens to provide a diagonal field of view and a depth of field comparable to the specification of a conventional colonoscope.

The functions of the colonoscope are activated via a hand controller with an ergonomic design connected to the robotic console ( $\triangleright$ Fig. 1c). It was designed to be operated by a single person. Directional control of the bending section is controlled by a precision joystick to replace the conventional manual double knobs. This robotic control not only simplifies the steering of the colonoscope but also reduces operator fatigue from prolonged procedures. All robotic control electronics, the pneumatic system as well as image processing functions, are embedded in a standalone wheeled console cart ( $\triangleright$ Fig. $1 \mathbf{d}$ ).

The level of procedural pain experienced by subjects undergoing the colonoscopy procedures was assessed using the Behavioural Pain Scale, Non-intubated (BPS-NI) [6,7] by the attending anesthetist. This pain score assessment evaluates three behavioral domains: facial expression, movement of upper limbs, and vocalization. Each domain contains four descriptors on a scale of 1 to 4 . The total score ranges from 3 to 12 , with 3 as no pain up to 12 which indicates most pain. The BPS-N1 measurements were repeated every 3 minutes after the initial measurement for the following five measurement stages: within approximately 2 minutes before insertion to the rectum, insertion from the rectum to cecum, withdrawal from cecum to the rectum, within approximately 2 minutes following removal of scope from rectum and at the start and end of applying abdominal pressure, if applied.

\section{Results}

Twenty subjects, 10 per study site, were recruited in the study. One subject in study site two failed to show up. A total of 19 subjects underwent a colonic examination using the NISInspire-C. Seventeen subjects had a complete colonoscopy procedure. At the discretion of the endoscopist, early discontinuation was observed in two subjects. All 19 subjects had a complete colonoscopy using conventional colonoscope. 

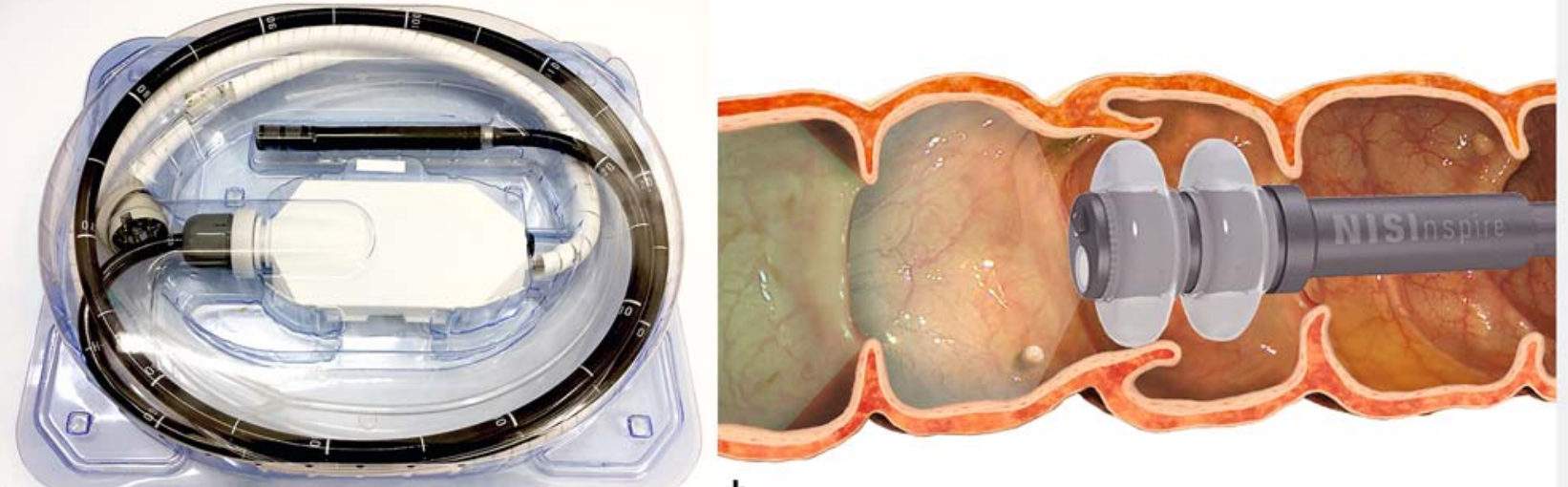

a

b
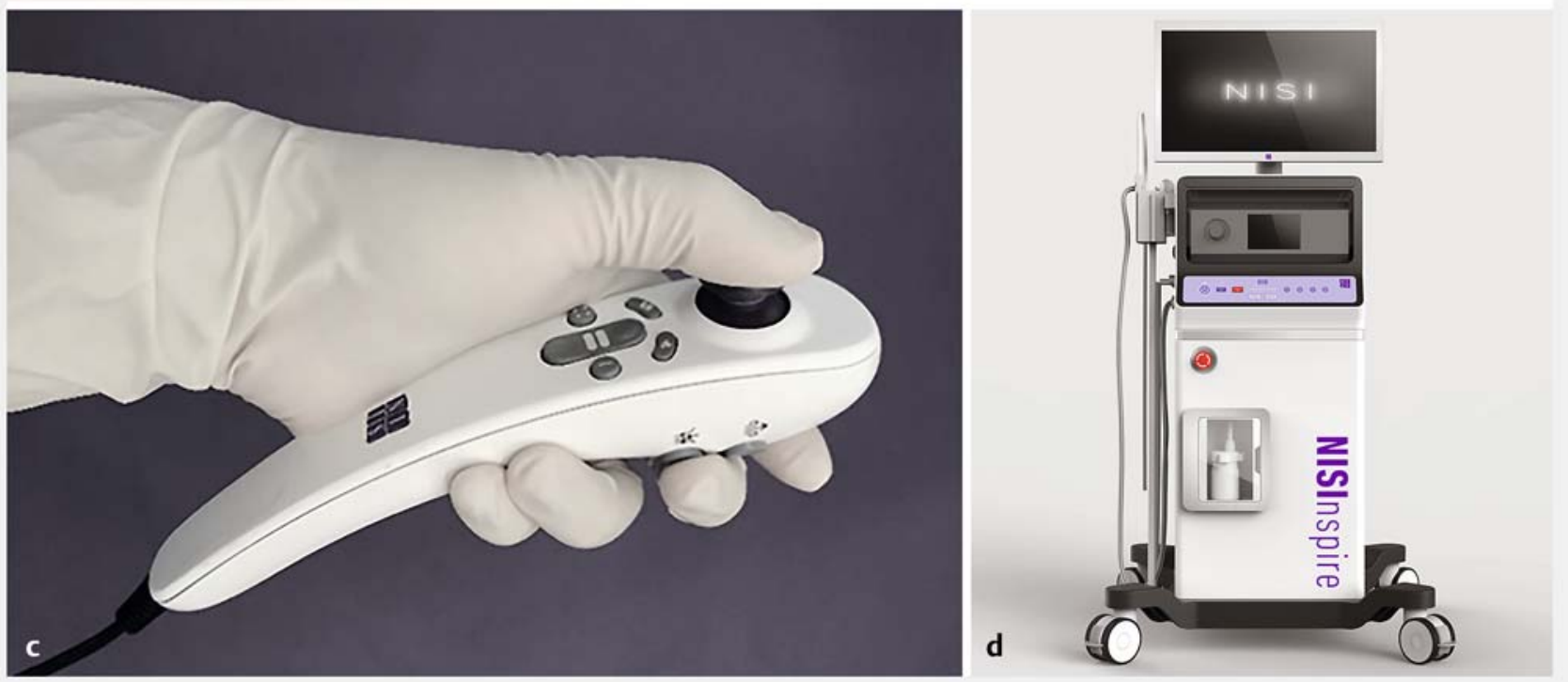

- Fig. 1 The NISInspire-C System. a NISInspire-C colonoscope. b Inflated twin balloons. c Hand controller. d The NISInspire-C console.

The demographics characteristics of the subjects are summarized in > Table 1. Fourteen subjects were male (73.7\%) and five were female (26.3\%). All subjects were Chinese with a mean age of 55.4 years (range: 40-69; SD: 8.5 years). Mean body weight and body mass index were $64.0 \mathrm{~kg}$ (range: 43.0-87.7; SD: $11.5 \mathrm{~kg}$ ) and $23.5 \mathrm{~kg} / \mathrm{m}^{2}$ (range: $17-33$; SD: $3.7 \mathrm{~kg} / \mathrm{m}^{2}$ ), respectively. All subjects received monitored anesthesia care. There were seven (36.9\%), nine (47.4\%) and three (15.8\%) subjects with BBPS 8-9, BBPS 6-7 and BBPS 3-5, respectively.

The CIRs were $89.5 \%$ (17/19) and the overall time-to-cecum (TTC) was 26.3 minutes (SD: 17.9 mins). The respective reasons for unsuccessful cecal intubation and, therefore, early discontinuation of the colonic procedure for the NISInspire-C were due to poor bowel preparation in one subject and severe sigmoid colon redundancy in another. The mean TTC for endoscopists $A$, B and C were $30.8 \pm 20.6$ minutes, $27.8 \pm 24.0$ minutes, and $21.3 \pm 10.5$ minutes, respectively.

Polyps were detected in seven of 19 subjects (36.8\%) during the NISInspire-C procedure. These were left to be removed by the conventional colonoscope. During tandem conventional colonoscopy, three more subjects were found to have adenomatous polyps. Complications including colonic perforation, bleeding, sedation-related issues and mucosal injuries from the balloon suction mechanism were not observed. The total BPS-NI values were measured for subjects who underwent the examination using the NISInspire-C System. The total BPS-NI values over the predefined measurement phases were quite steady with only scanty variations ( $\vee$ Table 2 ). The median total BPS-NI values were three $(I Q R=0.0-0.3)$ in any measurement phases during the NISInspire-C procedure. Abdominal pressure was applied to two subjects during the colonoscopy procedures.

\section{Discussion}

Despite enormous advances in camera optics and algorithms for enhanced detection, it is surprising how little in the basic mechanical design of conventional colonoscopes have changed in the last 40 years [8]. 


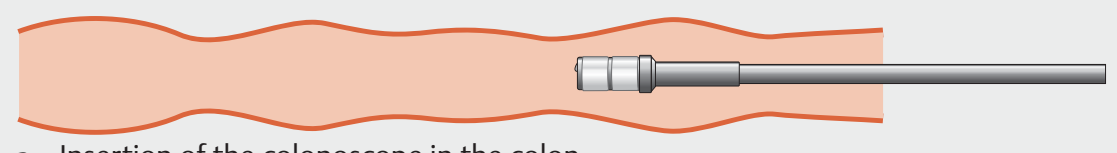

a Insertion of the colonoscope in the colon

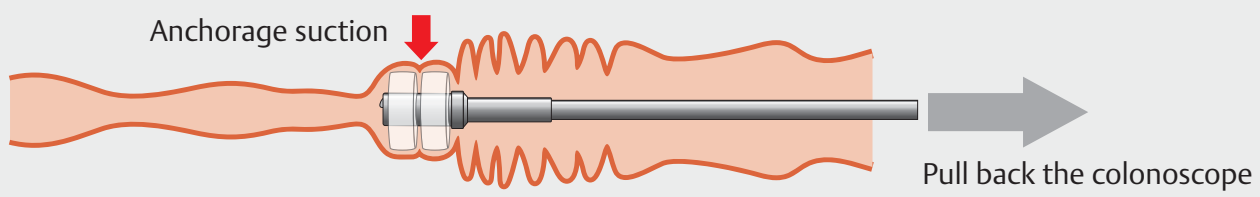

b Activation of balloon anchorage mechanism

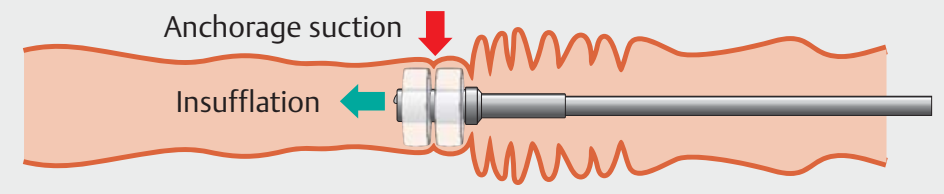

c Insufflate to open up luminal view

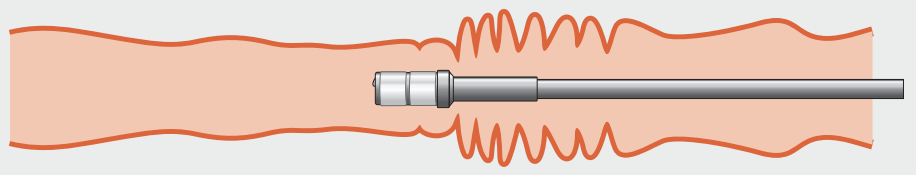

d Deactivation of balloon anchorage mechanism

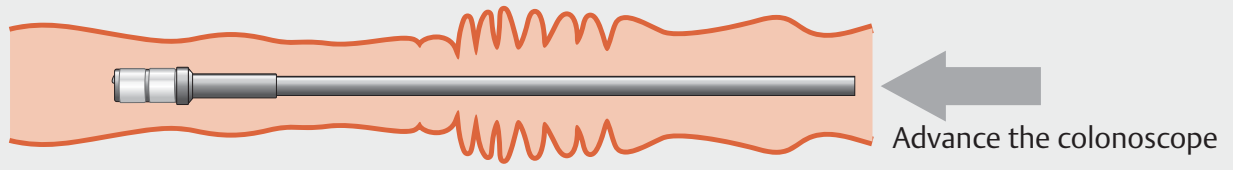

e Advancement ot the colonoscope in the colon

- Fig. 2 Mechanism of locomotion of the NISInspire-C colonoscope.

The NISInspire-C uses a twin-balloon anchorage mechanism that can allow a reliable and secure anchor of the colonoscope to the colonic wall to enable straightening the colon and uses an alternative locomotion mechanism that does not solely rely on the surgeon's push to advance in the colon. It is also designed to improve the ergonomics of the operating endoscopist. The light-weight, all-in-one electronic hand controller is designed to provide ergonomic handling to reduce physical strain and the risk of injuries associated with repetitive turning of tight knobs.

A rising concern is the possibility of transmitting infectious diseases through reusable endoscopes or ineffective device reprocessing [9-11]. A recent study reported data collected from ambulatory surgery centers on post-endoscopic procedure infection, a rate of infection $\leq 7$ days after the procedure just slightly higher than 1 in 1,000 for screening colonoscopies and approximately 1.6 per 1,000 for non-screening colonoscopies which were previously believed to be approximately 1 in 1 million [12]. It has been reported that microbial growth was detected in $71 \%$ of endoscopes, even though standard reprocessing procedures were used [9]. The demand for a single-use colonoscope is set to increase amid the COVID-19 crisis. Coronavirus has been found to persist in fecal samples of COVID-19 patients even though clearance in the respiratory tract was confirmed [13]. The study suggested that disposable endoscopes could be considered for patients at high risk of acquiring or transmitting infections.

Reprocessing of colonoscopes is associated with significant cost. The reprocessing cost, including cleaning supplies and labor cost, is in the range of USD\$100 to USD \$200 per endoscope, according to a market survey. In the U.S. market, the true cost of using a reusable endoscope, including its pre-procedure capital cost, repair cost, cleaning cost, and infectionrelated treatment cost, is over USD $\$ 500$ per procedure. The use of a disposable endoscope for colonoscopy could be envisaged to be more cost-effective than the conventional, reusable counterparts.

The TTC varied among the three endoscopists. One possible reason to explain this was that they were at different stages of their learning curve at the start of the study. One of the endoscopists had been involved with the development and testing of the prototypes of the NISInspire-C and, therefore, was more familiar with the handling of the instruments. The remaining two endoscopists familiarized themselves with the NISInsipre-C using a porcine intestine simulator. The learning curve for trained endoscopists was estimated to be between five to 10 cases.

The examination was terminated in one subject due to colon tortuosity. The balloon anchorage mechanism was found to be working. The reason as to why the tortuosity was not overcome was determined afterward. The team attributed this to the in- 
- Table 1 Demographics and baseline variables.

\begin{tabular}{|l|l|l|}
\hline Variable & & N=19 \\
\hline Age (years) & Mean (SD) & $55(8.5)$ \\
\hline Gender, N (\%) & Female & $5(26.3 \%)$ \\
\hline Race and ethnicity, N (\%) & Male & $14(73.7 \%)$ \\
\hline Bodyweight $(\mathrm{kg})$ & Asian-Chinese & $19(100.0 \%)$ \\
\hline BMI (kg/m $\left.{ }^{2}\right)$ & Mean (SD) & $64(11.5)$ \\
\hline Monitored anesthesia care performed? N (\%) & Mean (SD) \\
\hline BMI, body mass index. & Yes & $24(3.7)$ \\
\hline
\end{tabular}

- Table 2 Summary of total behavioral pain scale, non-intubated (BPS-NI) value.

\begin{tabular}{|c|c|c|}
\hline \multirow[b]{2}{*}{ Measurement phase ${ }^{1}$} & \multicolumn{2}{|l|}{ Colonoscope } \\
\hline & NISInspire- $C^{2}$ & Conventional ${ }^{3}$ \\
\hline \multicolumn{3}{|c|}{ Within approximately 2 mins before insertion to rectum } \\
\hline$\cdot \mathrm{N}$ & 18 & 18 \\
\hline - Mean (SD) & $3.0(0.00)$ & $3.0(0.00)$ \\
\hline - Median (Q1, Q3) & $3.0(3.0,3.0)$ & $3.0(3.0,3.0)$ \\
\hline " Min, Max & $3.0,3.0$ & $3.0,3.0$ \\
\hline \multicolumn{3}{|c|}{ Insertion from rectum to cecum } \\
\hline$\cdot N$ & 18 & 18 \\
\hline - Mean (SD) & $3.3(0.53)$ & $3.1(0.24)$ \\
\hline - Median (Q1, Q3) & $3.0(3.0,3.3)$ & $3.0(3.0,3.0)$ \\
\hline " Min, Max & $3.0,4.8$ & $3.0,4.0$ \\
\hline \multicolumn{3}{|c|}{ Withdrawal from cecum to rectum } \\
\hline$\cdot \mathrm{N}$ & 17 & 18 \\
\hline - Mean (SD) & $3.0(0.00)$ & $3.1(0.15)$ \\
\hline - Median (Q1, Q3) & $3.0(3.0,3.0)$ & $3.0(3.0,3.0)$ \\
\hline - Min, Max & $3.0,3.0$ & $3.0,3.6$ \\
\hline \multicolumn{3}{|c|}{ Within approximately 2 mins following removal of scope from rectum } \\
\hline$\cdot \mathrm{N}$ & 17 & 18 \\
\hline - Mean (SD) & $3.0(0.00)$ & $3.0(0.00)$ \\
\hline - Median (Q1, Q3) & $3.0(3.0,3.0)$ & $3.0(3.0,3.0)$ \\
\hline - Min, Max & $3.0,3.0$ & $3.0,3.0$ \\
\hline
\end{tabular}

crease in length between the tip of the scope and the bending section, in order to accommodate the twin balloons, reducing the ability of the scope to overcome sharp bends. This was also believed to be one of the reasons, apart from the learning curve for a new device, why the TTC was relatively long for experienced endoscopists. Also, the endoscopists felt there was a need to advance the scope manually despite the balloon suction mechanism. It is envisaged that future modifications will 
focus on addressing this and hopefully would reduce manual advancement of scope to a minimum.

\section{Conclusion}

This study demonstrated that the NISInspire-C is feasible with overall results showing that CIRs with it appear realistic. The device is single-use to eliminate any risks of cross-infections between patients. It can also eliminate reprocessing and, therefore, reduce costs incurred.

\section{Competing interests}

Dr. Foo is an advisory member for NISI (HK) Ltd. Dr. Leung is an advisory member for NISI (HK) Ltd and consultant for Medtronic. Dr. Yeung is the Chairman \& CTO of Bio-Medical Engineering (HK) Limited (subsidiary company of NISI (HK) Ltd). Drs. Cheung, Lam, and Sreedhar are employees of Bio-Medical Engineering (HK) Limited.

\section{References}

[1] González-Candelas F, Guiral S, Carbó R et al. Patient-to-patient transmission of hepatitis $\mathrm{C}$ virus (HCV) during colonoscopy diagnosis. Virol J 2010; 7: 217

[2] Kovaleva J, Peters FT, van der Mei HC et al. Transmission of infection by flexible gastrointestinal endoscopy and bronchoscopy. Clin Microbiol Rev 2013; 26: 231-254

[3] Lai E], Calderwood AH, Doros G et al. The Boston bowel preparation scale: a valid and reliable instrument for colonoscopy-oriented research. Gastrointest Endosc 2009; 69: 620-625
[4] Rex DK, Schoenfeld PS, Cohen J et al. Quality indicators for colonoscopy. Gastrointest Endosc 2015; 81: 31-53

[5] Yeung CK, Cheung JL, Sreedhar B. Emerging next-generation robotic colonoscopy systems towards painless colonoscopy. J Dig Dis 2019; 20: 196-205

[6] Chanques G, Payen JF, Mercier $G$ et al. Assessing pain in non-intubated critically ill patients unable to self report: an adaptation of the Behavioral Pain Scale. Intensive Care Med 2009; 335: 2060-2067

[7] Payen JF, Bru O, Bosson JL et al. Assessing pain in critically ill sedated patients by using a behavioral pain scale. Crit Care Med 2001; 29: 2258-2263

[8] Shergill AK, McQuaid KR. Ergonomic endoscopy: An oxymoron or realistic goal? Gastrointest Endosc 2019; 90: 966-970

[9] Ofstead CL, Wetzler HP, Heymann OL et al. Longitudinal assessment of reprocessing effectiveness for colonoscopes and gastroscopes: Results of visual inspections, biochemical markers, and microbial cultures. Am J Infect Control 2017; 45: e26-e33

[10] Neves MS, da Silva MG, Ventura GM et al. Effectiveness of current disinfection procedures against biofilm on contaminated $\mathrm{Gl}$ endoscopes. Gastrointest Endosc 2016; 83: 944-953

[11] Ofstead CL, Heymann OL, Quick MR et al. Residual moisture and waterborne pathogens inside flexible endoscopes: Evidence from a multisite study of endoscope drying effectiveness. Am J Infect Control 2018; 46: 689-696

[12] Wang P, Xu T, Ngamruengphong $S$ et al. Rates of infection after colonoscopy and osophagogastroduodenoscopy in ambulatory surgery centres in the USA. Gut 2018; 67: 1626-1636

[13] Lui RN, Wong SH, Sánchez-Luna SA et al. Overview of guidance for endoscopy during the coronavirus disease 2019 pandemic. J Gastroenterol Hepatol 2020; 35: 749-759 